\title{
THE BROKEN NOSE
}

\author{
By JACK PENN, F.R.C.S. \\ Frofessor of Plastic and Maxillo-Facial Surgery, University of the Witwatersrand, Fohannesburg
}

With increased facilities for sport, frequency of industrial accidents and motor collisions, nasal fractures are increasing in number, both in men and women. Frequently, too, the injury occurs in children, and it is often undiagnosed because the nasal bones have not fully developed, but the septum may be deviated or crumpled, giving rise to gross deformity of the nose when it does develop. Considering the number of people who have at some time in their lives broken their noses without having adequate treatment afforded them, it is not surprising to note the high percentage of respiratory troubles existing today associated with a deflection of the septum, all of which could have been avoided if in the first instance correct treatment had been carried out.

The function of the nose in relation to bodily and mental health has been greatly underestimated in the past, for an obstructed airway which causes difficulty in breathing, snoring when asleep, and which upsets the timbre of the voice, is a condition which affects the whole personality of the patient. Not the least important factor is that the nose, being in the middle of the face, has the focus of attention and deviations from normal are always noticeable. Probably more complexes are set up by nasal deformities than by any other. Some individuals consider it an asset to look ' tough' as it adds to the brutal appearance of a boxer or a soldier, but in everyday life where competition is keen and where personality counts for so much, normalcy is extremely important.

\section{Etiology}

Although sport accounts for the majority of broken noses in men, the frequency of industrial accidents and motor collisions has increased the proportion of nasal fractures in women. Frequently the direction of force is from one side or the other, deviating the nose to the opposite side (Fig. I). Sometimes the blow is head on, in which case the nasal complex is crushed backwards into the nasal fossa, constituting the first stage of the so-called mid-third facial crash (Fig. 2).

It may often be associated with a fracture of the maxilla, so that the entire block of the nasomaxillary bones is pushed back towards the ethmoid region (Fig. 3). The amount of displace- ment in either case is proportionate to the force, but sometimes the external nose may not be displaced when examined, as it may spring back into its original position. The septum, however, may still be deviated.

Diagnosis of a broken nose should never be made from external examination only. It must be recalled that most of the structure making up the nasal framework, as well as that of the septum, is cartilaginous. Only the tiny nasal bones, the perpendicular plate of the ethmoid and the vomer constitute the bony elements. Many massive deviations are associated with displacement of one cartilage on another, or on the nasal bones.

It will therefore be obvious that the use of $\mathrm{X}$-rays in diagnosis is extremely limited. Diagnosis should depend upon clinical examination only. The presence of a deviation, or extreme swelling or change of contour associated with haemorrhage, or even the presence of haemorrhage alone following trauma is sufficient to warrant diagnosis of a fractured nose. Gentle manipulation may elicit crepitus; if carefully done this can be carried out without causing pain.

\section{Treatment of the Recent Fracture}

This should be carried out as soon as possible, but may be effective up to six weeks after the development of the deformity. In cases where the nose is still numb, e.g., following injury on the football field, it is possible to correct the position by grasping the nasal bridge between the finger and thumb and tweaking it into position. If, however, it is suspectcd that the septum is affected, treatment in the operating theatre is essential.

Anaesthesia may be either local or general. General anaesthesia should consist of full intubation, but never pentothal alone, for haemorrhage follows manipulation and this may upset the airway in a case where the throat has not been packed off, and may result in danger to the unconscious patient by the entrance of blood into the trachea.

Manipulation and splintage under local anaesthesia is, however, fully effective. The patient is premedicated by nembutal 3 gr. one and a half hours before operation, and $\frac{1}{3}$ gr. of omnopon half an hour before. The nose is then packed with 5 
per cent. cocaine on each side of the septum. Infiltration of the subcutaneous tissues with 2 per cent. novocaine and adrenalin is carried out, the injection being placed between the upper and lower cartilages on each side.

A left-sided Walsham forceps is then placed in position so that the inner blade is passed along the under-surface of the nasal bones on that side, while a padded outer blade grasps the outer aspect (Fig. 4). Manipulation disimpacts the outer bones and displaced cartilages so that they may be brought into their normal positions. The right side is treated similorly.

Finally, an Ashe's forceps is inserted vertically so that the blades pass along either side of the septum, along the floor of the nose (Fig. 5). They are brought forward by a circular movement so that the septum is ' milked' into normal position. The fingers now test the modelling of the nose to see whether any spurs are obvious, and a plaster of Paris splint is applied.

There is far less bleeding with local anaesthesia than when a general anaesthetic is used, but if blood is accumulating at the back of the throat the patient may be allowed to expectorate this on to a sterile swab.

The plaster splint is cut (Fig. 6). It consists of seven layers of plaster of Paris, moistened in water and placed over the nose so that the T-piece is over the forehead, the vertical bar on the nose and the spread base is over the alae of the nose. While still moist the plaster is moulded into position, the bridge being grasped firmly between the two forefingers and thumbs and held there while the plaster hardens. This reduces oedema and preserves the shape. The infiltration of novocaine does not affect it in any way. When the plaster has hardened and lies snugly in position, scotch tape may be placed across the T-piece and base and also crossed over the bridge (Fig. 7). It is not necessary to plug the nose ; in fact this should be avoided for internal splintage plays no part in the treatment of the fractured nose as it is irritant, unnecessary and probably harmful. The splint may be left on for 48 hours, or longer if there has been much bruising, after which time it should be removed.

\section{Treatment of the Old Fracture}

I. The Deviated Nose. A fractured nose, if not manipulated into correct position at an early stage, causes disabilities which are both functional and cosmetic. Deviation of the nasal framework and the septum may cause obstruction to one or both sides, with all its attendant complications such as bad aeration, snoring, chronic catarrh, sinusitis, etc., as well as speech disabilities.
If the nose is bent but the airway is satisfactor it may be sufficient to straighten the nose from the point of view of appearance, but if the airway is net satisfactory it is necessary to improve it by carrying out a submucous resection.

An accurate assessment must be made of the deviation affecting (a) the upper third of the nose i.e. the nasal bones; (b) the middle third of the nose, i.e. the upper lateral cartilages together wit the cartilaginous septum; and (c) the lower thind of the nose, lower lateral cartilages and the res lationship of the columella to the membranous septum.

As with the recent fracture, X-rays are seldow of value for vast displacements may occur due dislocation of cartilage rather than to fracture off bone. This applies particularly if the septum is warped.

The anaesthetic of choice is local anaesthesi which infiltrates not only into the nasal framework as described above, but also into each side of tho nasal septum.

A curved incision is made on the membranous septum and the mucous membrane is dissected of the septum. This is done fully and widely in evero direction, particularly along the floor of the nose for it is here that obstruction interferes with aeration. The cartilage is then incised an 8 similar submucous dissection is made on the ofer side of the septum (Fig. 8). A septal speculurit then inserted, and the septum inspected, the obstruction is noted and the portion of septur which is bent or warped is removed. One mus be careful that sufficient cartilage should be le along the bridge of the nose to prevent collapse of the bridge, if and when a hump reduction necessary.

In some cases a submucous resection is sufficie to allow manipulation of the nose into a centrat position, but in others further treatment necessary. If a hump is present, this can be taken. off at an angle, the new profile line is trimmed, and. the columella is attached to its correct positiong after having reduced the lower segment of the septum which in many cases is dislocated into one nostril (Fig. 9).

In cases of extreme dislocation of the septurm into the nostril, it may be necessary to remove eve this portion of the septum and to reconstituto support for the columella by inserting a straighto portion of cartilage between the layers of mucous membrane as a free graft. It is usually possible to obtain an adequate strut of this sort from among the fragments of nasal septum which have just been resected.

An infracture of the frontal processes of the maxillae is now necessary. Usually the process is bilateral, but in some cases it is sufficient to counte 

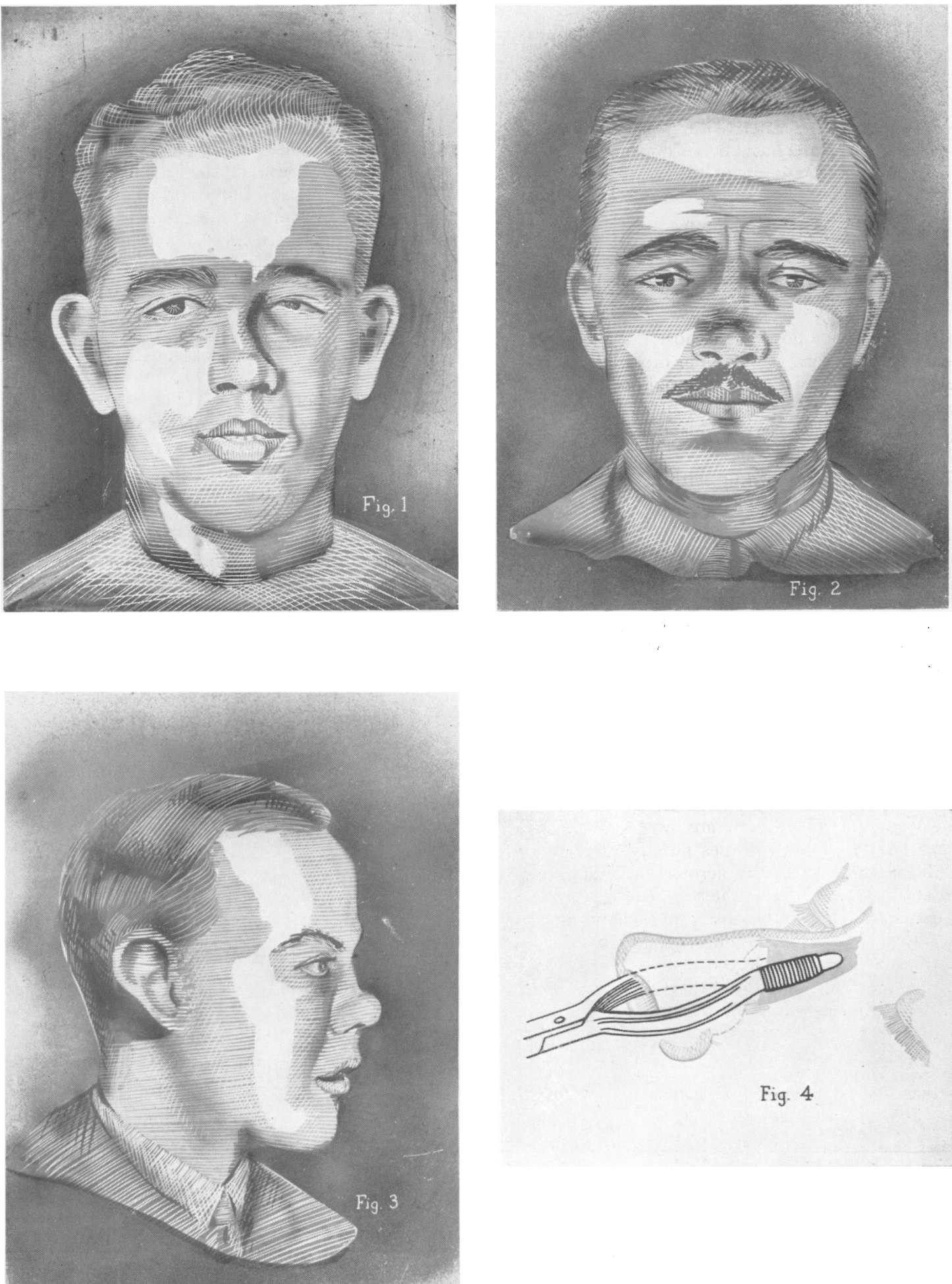

Fig. 4 


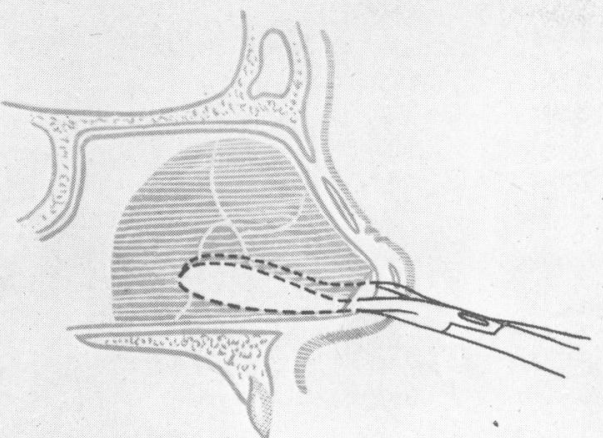

Fig. 5.

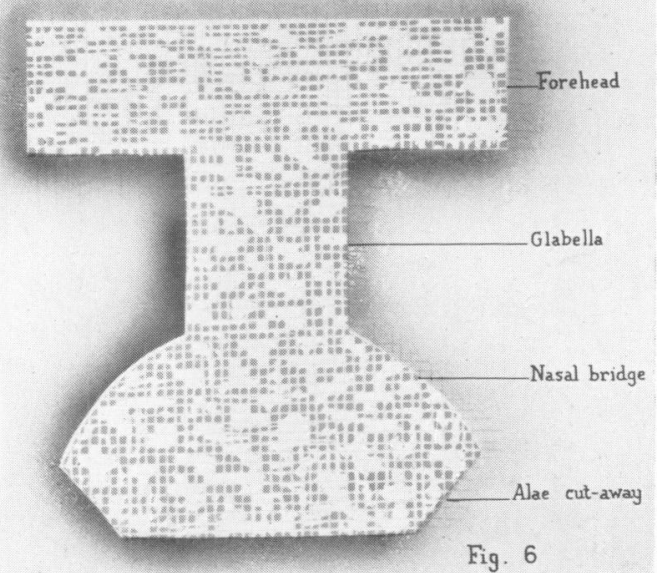

Fig. 6
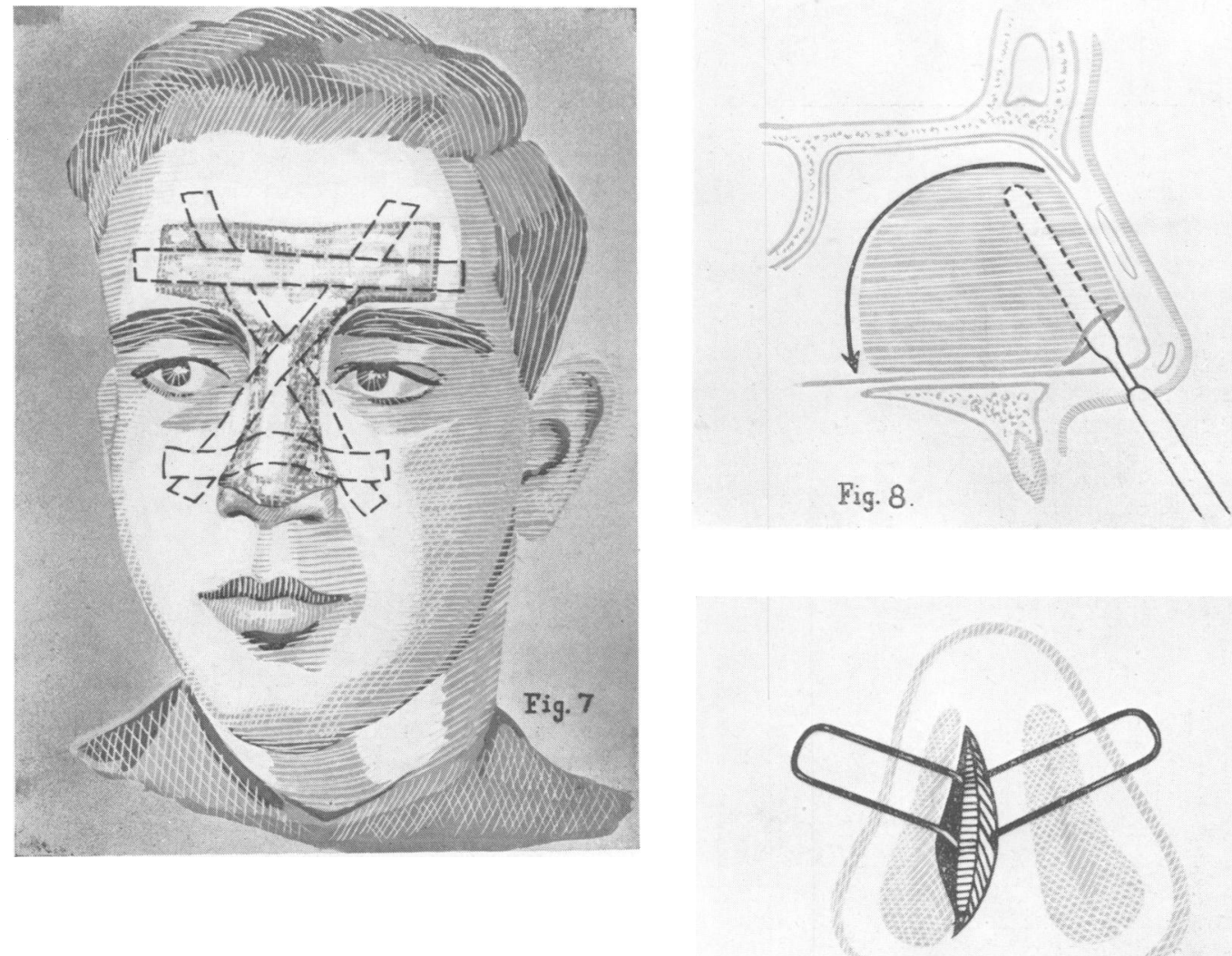

Fig. 9. 

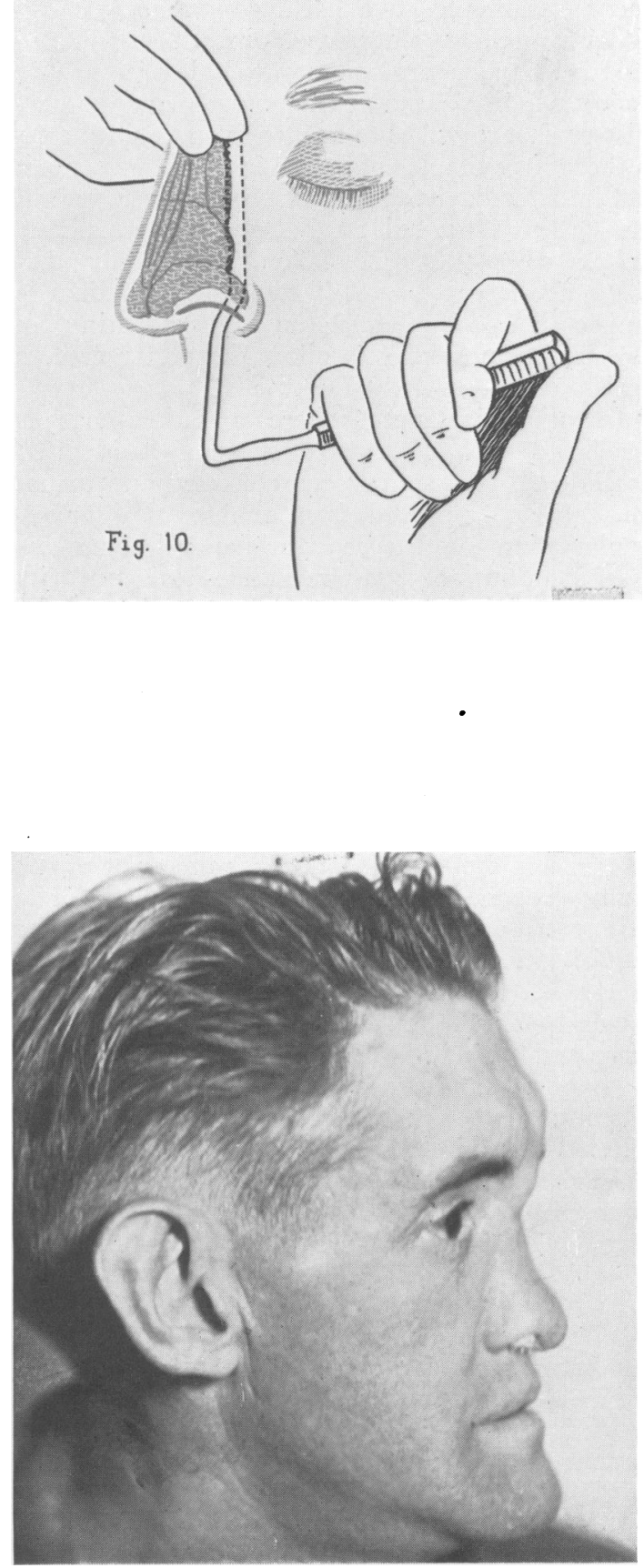

Fig. 12
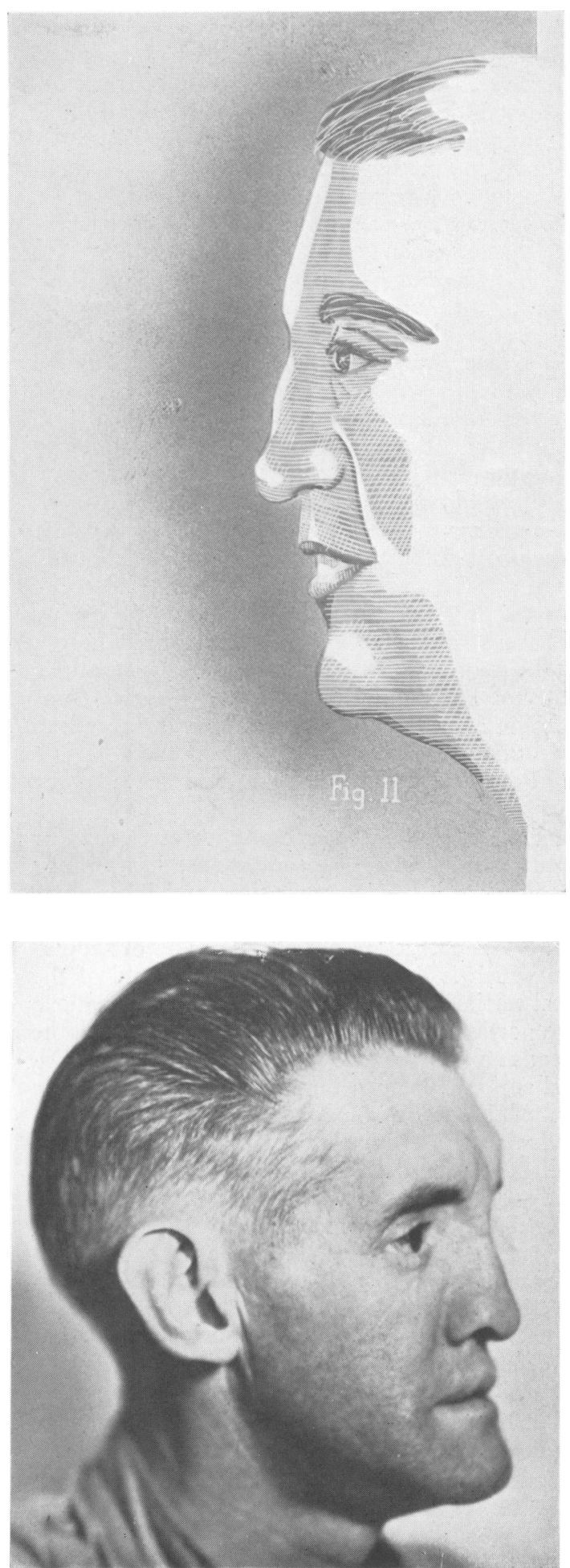

Fig. 13 
the deviation of the nose by performing a unilateral infracture on the convex side.

The next procedure is to assess whether one or both the lower lateral cartilages should be trimmed. In old-standing cases distortion of one or both of the lower lateral cartilages is usually evident.

Finally the plaster of Paris splint is applied, and if a submucous resection has been performed the nasal cavities are packed for 48 hours to prevent septal haematoma.

2. The Crushed Nose (Saddle-nose). The headon crash, as in plane or car collisions, is frequently associated with an impaction of the central mass of the face into the cavities of the nasal and ethmoid regions. When the force is not excessive a saddle-nose results, due to comminution and fracture of the nasal bones associated with crumbling and collapse of the septal cartilage. When the force is greater, the frontal processes of the maxillae may also be involved so that the entire walls of the antra are driven inwards, giving rise to a dishface deformity.

In either event the first essential is to elevate the nasal structure to its normal position where it may be kept, as previously shown, by means of a plaster of Paris splint, or in cases of severe comminution a through-and-through suture over lead plates on each side of the bridge of the nose is a useful method of preventing a recurrence of the collapsed bridge.

A great number of patients, however, come for treatment only when the saddle-nose has been in existence for a considerable time and is fixed by callus, so that manipulation is impossible.

Into this category also falls the type of saddlenose which is due to the surgical removal of the septal cartilage. Although it is possible to remove large portions of the deviated septum between the mucous layers in such a way as not to interfere with. nasal support, it must be recalled that the middle third of the nose is supported by the upper lateral cartilages which are virtually part of the septal cartilage, whilst the lower third of the nose, consisting of the alar cartilages together with the columella cartilage which forms the tip of the nose, is dependent for its support on the lower margin of the cartilaginous septum. Any interference with the profile line of the septum or with its lower edge abutting on the membranous septum will cause a collapse, not only of the bridge of the nose but also of the tip (Fig. I I).

The treatment in either case is to support the nose by means of some form of graft. The graft used may be of bone (Fig. 12), of cartilage (Fig. I3) or of a synthetic substance such as tantalum, acrylic, etc. In the majority of cases synthetic substances are not advisable, for sufficient autogenous grafts are easily obtainable. Homologous grafts of cartilage or even cadaver cartilage have been used. The disadvantages of synthetic sub $\frac{\Phi}{2}$ stances is that they do not round off, and sube sequently may cause irritation at certain points of stress. The disadvantage of cadaver cartilage i\& that it may be invaded by fibrous tissue to such ar extent that fracture and absorption is likely te. occur. Although live cartilage can be used $\frac{\bar{D}}{\vec{D}}$ either from the patient himself or from elsewhere $\stackrel{\Phi}{\triangle}$ the tendency for cartilage to warp unless previousl $\xi_{5}$ boiled is a disadvantage which may spoil an otherwise perfect result. Bone taken from the iliac crest. is the most suitable substance in every way, for it can be adequately shaped and becomes a part o. the patient's normal structure, as it frequentlo unites with subjacent bone. The only dis? advantage is that hospitalization of the patient is longer than with other methods, necessitating terin days in bed in order to allow the donor area tọ heal.

In other instances where a saddle-nose is associated with a deviated septum, it is ofterp possible to do a submucous resection and to use the cartilage removed from the septum for re implantation into the nasal bridge and columellao

In some instances where saddle-nose concavity is very small, for purposes of cosmesis and support it may be possible to obtain adequarte cartilage locally from the upper or lower laterat cartilages, and to implant them to fill in the formity in the profile line.

There is no doubt that if bone is decided uporz as a medium for support it should be obtaineof from the iliac crest. The approach is by means of an incision about 2 in. long over the crest of the्छ ilium, deepened until the crest is reached. The periosteum is stripped for about $I \frac{1}{2}$ in. and the requisite amount of bone is removed with an. osteotome. The bone is then shaped. It is usually about $1 \frac{1}{4}$ in. long, one end being fairl sharp and flat in order that it may be wedged undes th $\epsilon$ periosteum of the nasal bones, to act as a form of splint. The approach to the nose is by a splip columella incision. It is not advisable to make an incision into the nose, owing to the impossibility of sterilizing that area. Following the incision between the two alar cartilages, a tunnel is. fashioned, using scissors between the two crura passing over the attachments of the upper laterah cartilages to the septum, and finally dissecting it\$ way between the periosteum of the nasal bones and the nasal bones themselves. The bed should not be made excessively large, but just large enough t\& contain the graft according to the shape and sizes previously planned. The graft is now placed in position, and it is useful to wedge it between the periosteum and the nasal bones by means of metal punch. 
In the majority of cases, even in saddle-nose associated with the collapse of the columella, this wedging is an adequate support for the nose, improving the airway considerably, but it could if necessary be supplemented by a columella strut, i.e. implantation of a sliver of bone about $x$ in. long between the lower end of the graft and the spine of the maxilla.

Whatever method may be used in the treatment of either recent or old fractures, nothing short of a satisfactory airway and a normal cosmetic result should satisfy. This is the least that should be expected from those whose duty it is to deal with the problem of the broken nose.

\section{Summary}

I. Fractures of the nose are becoming more common both in men and in women. They may cause a syndrome of discomfort, deformity, disease, depression and even divorce.

2. Types of fractures sustained are illustrated.

3. Treatment of the recent fracture is detailed.

4. Treatment of the old fracture is discussed in relation to both the deviated and the crushed nose.

\section{RUTHIN CASTLE, NORTH WALES}

A Clinic for the diagnosis and treatment of Internal Diseases (except Mental or Infectious Diseases). The Clinic is provided with a staff of doctors, technicians and nurses.

The surroundings are beautiful. The climate is mild. There is central heating throughout. The annual rainfall is 30.5 inches, that is, less than the average for England.

The Fees are inclusive and vary according to the room occupied.

For particulars apply to THE SECRETARY, Ruthin Castle, North Wales.

Telograms : Castle, Ruthin.

Telephone : Ruchin 66 